\title{
A Framework for Analysing Sustainability by Using the Rewriting System
}

\author{
Kotaro Kakimoto and Toshiharu Taura \\ Graduate School of Science and Technology \\ E-mail:kakimoto@mi-5.scitec.kobe-u.ac.jp,taura@mech.kobe-u.ac.jp
}

\begin{abstract}
The social system must be transformed to a sustainable cycle-oriented social system immediately. Numerous studies have been conducted on environmental problems. Those studies, however, focused on forming a sustainable society from the top down, based on the belief that strong restrictions such as laws will lead to the development of a sustainable society. We consider this belief to be suspect and believe it is more effective to form a social system from the bottom up with retaining the current diversity of activities. In this paper, therefore, we discuss the nature of a cycle-oriented social system formed from the bottom up to achieve a sustainable society. By analysing its nature, we can identify the conditions under which the system will be sustainable. In order to conduct this analysis, we apply the abstract rewriting system on multisets (ARMS) technique. In this paper, we propose a framework for analysing sustainability using ARMS.
\end{abstract}

\section{Introduction}

Environmental problems have been receiving increasing attention and we have been facing them. Therefore our social system must be changed to a sustainable cycle-oriented social system immediately[1,2]. A considerable number of studies have been conducted on this subject. However those studies tend to regard the cycle-oriented social system as a very simple concept. We believe that it is important to take a closer look at how we should form the cycle-oriented social system and what it basically is.

Electric Household Appliances are being enforced in Japan, so that we must bring televisions, washing machines, refrigerators and air conditioners to specific manufacturers and pay them the cost, whenever we want to discard them. It seems that we have tacitly agreed that our activities should be restricted and that this is necessary, in order to protect the environment and form a sustainable society by applying strong restrictions from the top down. Yet consumers could reuse items they are going to discard in another way by using their imagination, if our activities were not strictly controlled. This will possibly have a vital impact on economic issues. Hence it may be more efficient to form a social system from the bottom up and retain a diversity of activities. Of course, this is not to say that we can do whatever we want to do. If we simply started to form a social system without any restrictions from the bottom up, it would end up causing other environmental problems such as those being faced by our present society and would not last for very long.

We believe that we need to analyse the cycle-oriented social system and then find appropriate conditions for achieving a sustainable system by means of computer simulation. Many studies have focused on optimising the material flow in a certain time section, on the basis of the belief that the pursuit of materials circulation will enable the development of a sustainable society. We consider this belief to be suspect, and consider that the basic analysis of aspects of the nature of the cycleoriented social system, such as sustainability, diversity of actions and the complexity of the system, should be investigated. In order to conduct such an analysis, we apply the technique of the abstract rewriting system on multisets (ARMS)[6]. In this paper, we propose a framework for analysing sustainability using ARMS. 


\section{Modelling of Cycle-Oriented So- ciety}

\subsection{Cycle-Oriented Social System}

As environmental problems are becoming more serious, laws and technical innovations are being enforced to form a sustainable society. However it is taken for granted that consumers' choices should be restricted. It appears that the social system is being formed from the top down. Generally speaking, in our society, producers add value to products by using their own imagination. In other words, they are resources for economic activity. On the other hand, in a cycle-oriented social system, producers as well as consumers can add value to products and reuse them, because consumers stand at the starting point of material circulation. For instance, it has been proposed that prosumers who produce things they want to use should appear in a sustainable society[3]. They will take an active part in the social system. As a result, if it is possible to utilise their contributions efficiently so as to form the social system, we believe that we can form a vital social system. What we mentioned means that we should form the cycle-oriented social system from the bottom up. Therefore, we must find a method for modelling the social system and to establish the system from the bottom up. We have used a rewriting system as a tool to model a social system formed from the bottom up[4].

\subsection{Viewpoints of Our Study}

We tend to associate a system from the bottom up with a complex system. However, we also assume that a great complex social system is not sustainable. In the complex system, there are numerous diverse activities, so that without any restrictions, the circulations of materials will collapse. What must be considered is that a very wide range of our diverse activities without any restrictions have clearly had a significant impact on the environment and have caused environmental problems which are an increasing cause for concern. Therefore, we need to find restrictions to make the system sustainable. On the other hand, we assume that a very simple social system cannot last long either. In the simple system, there are only a few activities, which means most activities are restricted. We expect that an appropriately diverse range of activities will make our society vital.

With respect to these viewpoints, we need to have a framework in which we can discuss correlation sustainability, the complexity of the system and the re- strictions required for the cycle-oriented social system which we are aiming at. The purpose of this study is to identify restrictions in order to make the system sustainable. When we use the rewriting system as a tool for modelling, it can deal with the termination property of calculations, the social system in an abstract manner and identify which restrictions influence the system.

\subsection{ARMS}

ARMS is similar to a chemical solution in a container where floating molecules can interact with each other according to reaction rules, and finite multisets of elements corresponding to molecules and reaction rules are specified by using rewriting rules. ARMS can deal with systems with many degrees of freedom and simulate the emergence of complex cycles such as chemical oscillations which are often found in the emergence of life[5]. Furthermore, the modelling and analysis of an ecological system by using ARMS are attempted[6].

ARMS is defined as

$$
\Gamma=(A, R)
$$

where $A$ denotes the types of elements in the multisets and $R$ denotes rewriting rules[6]. Here we show how ARMS works. An ARMS is defined as

$$
\begin{aligned}
\Gamma= & (A, R) \\
A= & \{a, b, c, d\} \\
R= & \left\{a, b, d \rightarrow c: r_{1}, b \rightarrow d: r_{2},\right. \\
& \left.a \rightarrow b, d: r_{3}, c \rightarrow b, d: r_{4}\right\} .
\end{aligned}
$$

In this example, we define the size of the multisets as 4 , the initial state in the multisets is $\{a, a, b, d\}$ and a rewriting rule is randomly selected in each step. One $a$ is set in the multisets in every step unless the total number of elements exceeds 4.

$$
\begin{array}{cl}
\{a, a, b, d\} & \\
\downarrow & r_{1} \text { is applied. } \\
\{a, a, c\} & \\
\downarrow & \\
\{a, b, c, d\} & r_{3} \text { is applied. } \\
\downarrow & \\
\{a, c, d, d\} & r_{2} \text { is applied. } \\
\downarrow & \\
\{a, c, d, d\} & r_{4} \text { is not } \text { applied. } \\
&
\end{array}
$$


First, in this example, $r_{1}$ is selected and can be applied because the total number of elements never exceeds 4 when $r_{1}$ is applied. Hence the state $\{a, a, b, d\}$ changes to $\{a, a, c\}$. Second, after $r_{3}$ and $r_{2}$ are selected and applied, the state becomes $\{a, b, c, d\}$. Here if $r_{4}$ is selected, it is never applied because the total number of elements exceeds 4 . It reaches the halt state, which means that the calculations have terminated, because there is no rule to apply[5]. When we apply this technique, we can discuss the termination property and deal with the social system abstractly.

\subsection{Rewriting Rules in the System}

In order to model the cycle-oriented social system by using ARMS, we regard a rewriting rule as an action which we usually perform in the social system. The society and circulatory materials are regarded as a certain container and various elements, respectively. We basically use three kinds of rewriting rules : Rule 1, Rule 2 and Rule 3(Fig.1). For instance, we define an ARMS as follows;

$$
\begin{aligned}
A= & \{a, b, c, d, e\} \\
R= & \left\{a, b, b \rightarrow c: r_{1}, c \rightarrow d, e, e: r_{2},\right. \\
& \left.d, d, e \rightarrow a, b, b: r_{3}\right\}
\end{aligned}
$$

$a$ and $b$ correspond to resources which are needed for production. $c$ corresponds to a product. $d$ and $e$ correspond to resources to be recycled.

$r_{1}$ denotes the process of production $c$ defined as Rule 1, as a product is assembled from various parts. $r_{2}$ denotes the process of consumption and resolution defined as Rule 2 , as a product is taken apart after it is bought from a store. $r_{3}$ denotes the process of recycling defined as Rule 3. A model, which we define as a cycle-oriented social system, is summarised in Fig.2. At the initial state, rewriting rules are generated randomly according to some restrictions as follows;

- In Rule 1, the left-hand side of the rewriting rule has some resources and the right-hand side has a product.

- In Rule 2, the left-hand side of the rewriting rule has a product and the right-hand side has some resources to be recycled.

- In Rule 3, the left-hand side of the rewriting rule has some resources to be recycled and the righthand side has some resources.
- In Rule 3, the number of elements on the lefthand side is equal to that on the right-hand side.

- Each rule needs a certain amount of energy for application which depends on the types of elements on the left-hand side of the rewriting rule.

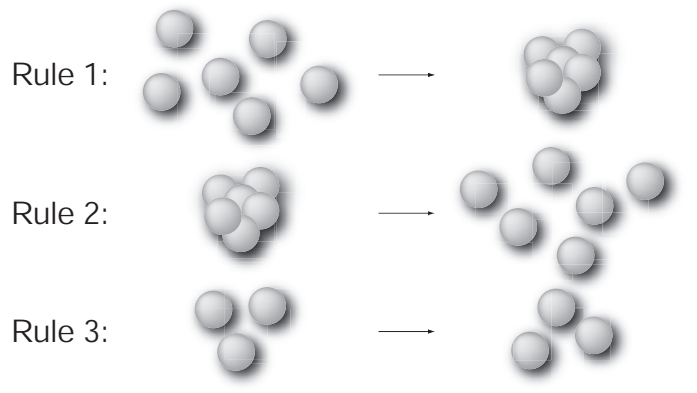

Fig. 1: Rewriting rules in the cycle-oriented social system

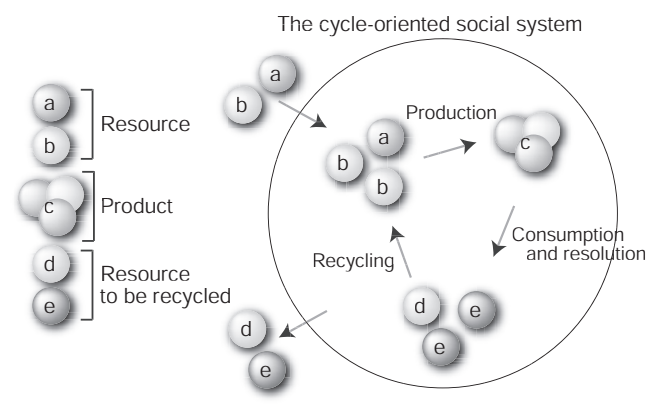

Fig. 2: A model of the cycle-oriented social system

However, we cannot deal with the dynamics of the social system if we simply regard circulatory processes as rewriting rules in ARMS. Here, we introduce a technique to ARMS. In order to deal with the dynamic social system, all rewriting rules are evaluated after a certain number of steps according to an evaluation function. A rule which is applied the maximum number of times among the rewriting rules copies itself to the group of rules, whereas rules which are not used for a certain number of steps disappear, so that rules which cannot adapt to changes in the environment are eliminated. In addition, a new rewriting rule is added after a certain number of steps, so that the group of rewriting rules remains diverse. We use a very simple evaluation function $F$ as follows; 


$$
F=W_{1} \times T+W_{2} \times c / E,
$$

where $T$ denotes the applied times, $E$ denotes the energy required for application, $W_{1}$ and $W_{2}$ denote coefficient values and $c$ is a constant. Using the evaluation function $F$ and the technique mentioned above, the system will obtain rules that are used frequently with small amount of energy to circulate elements, and the system can thus develop the rules and form from the bottom up.

\section{Simulation}

We develop the cycle-oriented social system by using ARMS in a computer. In this simulation, we observe the behaviour of the system made up from the bottom up and determine conditions under which the system can obtain many rules corresponding to our actions; we assume that diverse actions in the system are effective. In nature, species population stability is typically greater in structurally complex communities than in simple ones[7]. Therefore, we describe the complexity of the system by using a number of different kinds of elements as a restriction. If there are various elements in the system, it yields a complex system. On the other hand, if there are only a few different kinds of elements, it yields a simple system. We simulate how changes in the number of rewriting rules are reflected in the behaviour of the system.

\subsection{Setup of Simulation}

In Case 1, Case 2 and Case 3, there are 6, 15 and 45 kinds of elements in the initial states, respectively. The size of the multisets is 10000 . The selection is performed every 250 steps and a new rewriting rule is introduced into a set of rules every 20 steps. An element which is selected randomly flows into the multisets every two steps. An element selected randomly flows out from the multisets every four steps.

\subsection{Results and Considerations in the Sim- ulation}

In Fig.3, Fig. 4 and Fig.5, the x-axes indicate the number of steps and the y-axes indicate the total number of rewriting rules in the systems. A step refers to a process in which a rewriting rule is selected and its application attempted. Fig.3, Fig.4 and Fig.5 show the transitions of the numbers of the rewriting rules in Case 1, Case 2 and Case 3, respectively.

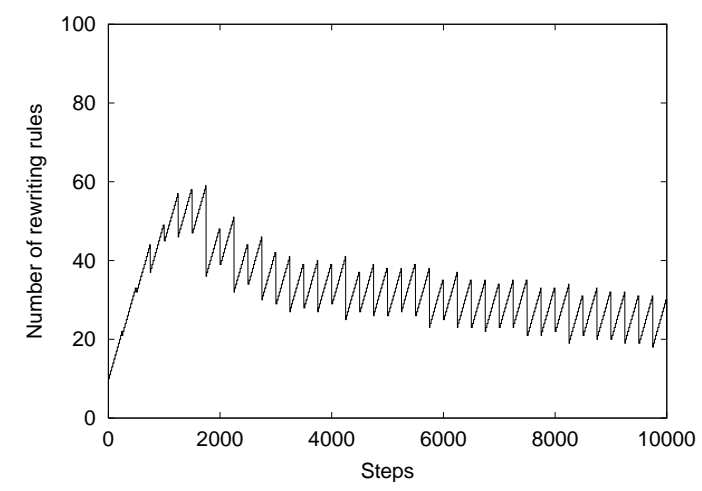

Fig. 3: Correlation between the number of rewriting rules and steps (Case 1 )

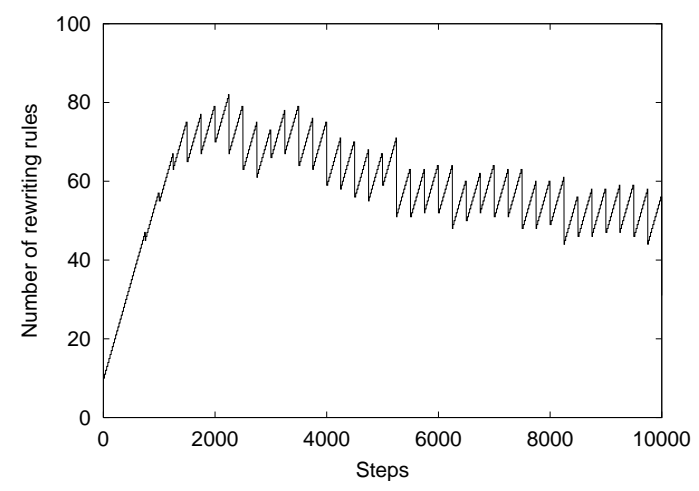

Fig. 4: Correlation between the number of rewriting rules and steps (Case 2)

In Fig.3, the number of rewriting rules did not change significantly, although it subtly oscillated. In this state, the number of rewriting rules did not change sharply and it is more stable than in Case 3 in Fig.5. In Fig.4, the number of rewriting rules was very high, compared with Case 1. However there was a point around the 5000th step where the number of rewriting rules changed sharply. In Fig.5, once the number of rewriting rules became high, it sometimes reduced significantly around the 3000th step and again at the 6000th step. It reached the lowest value in all Cases around the 10000th step. It was found from these results that the complex cycleoriented system could obtain a higher number of the rules corresponding to our actions than the simple system. However the greater complexity of the system did not allow it to reach a stable state. 


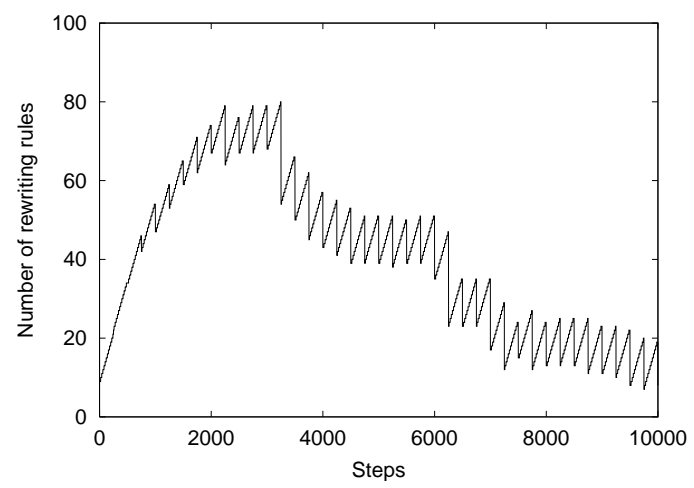

Fig. 5: Correlation between the number of rewriting rules and steps (Case 3 )

Next, we show the result that for a simulation performed 400 times for each Case. In Fig.6, the x-axis indicates the number of Cases and the $\mathrm{y}$-axis indicates the average number of rewriting rules. Case 2 has the highest number of rewriting rules and Case 1 had the lowest one. This result shows that it is not possible to obtain many rewriting rules simply by adding various elements to the system.

We see the average numbers of rewriting rules of Case 1, Case 2 and Case 3 in Fig.7. The $x$-axis indicates the number of Cases and the $y$-axis indicates the average number of steps needed for termination. In Case 2, it was found from the result that the system sustained for the longest steps in Cases, whereas in Case 3, the system kept circulating longer than Case 1. Complex cycle-oriented social systems, where there are more actions than simple ones, can make the life of the system longer than in simple systems. However, if we make the system complex without restrictions, as in Case 3, the system does not last any longer.

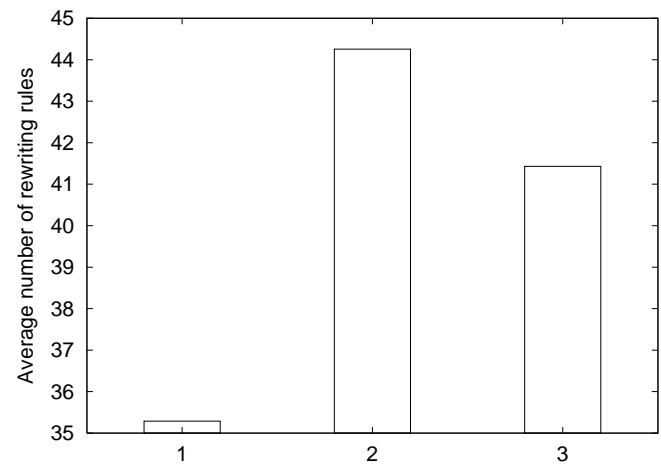

Fig. 6: Average number of rewriting rules

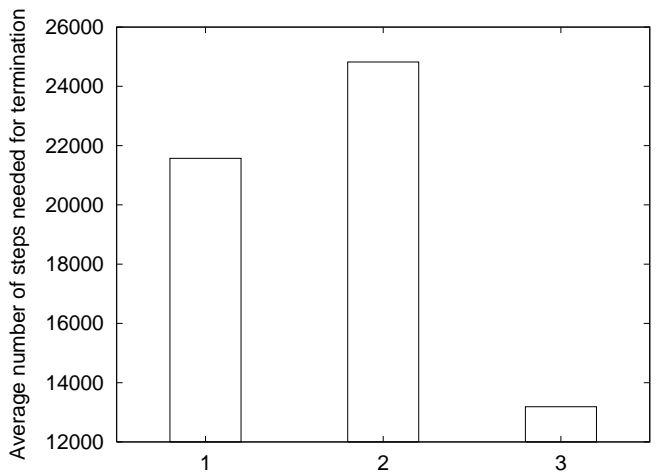

Fig. 7: Average number of steps needed for termination

Through the simulations, we found that sharp fluctuations of the number of rewriting rules occurred sometimes. In order to determine the reason for this, we performed another simulation. Fig.8 shows that the correlation between the number of elements and the number of times that the number of rewriting rules changed sharply. In Fig.8, the x-axis indicates the number of elements in the system and the $y$-axis indicates the number of times that the number of rewriting rules changed sharply.

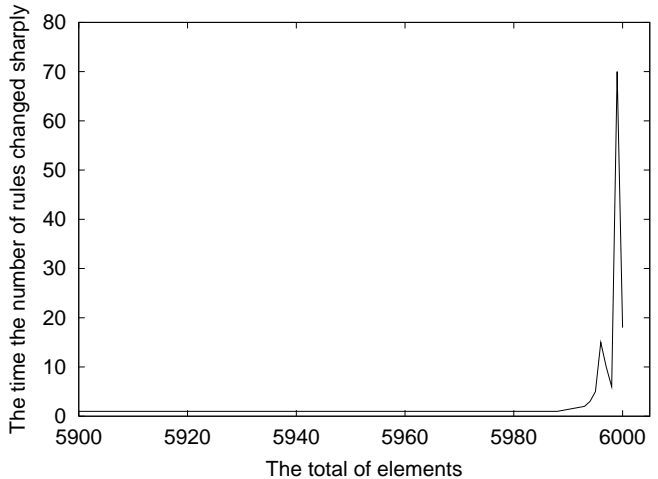

Fig. 8: Correlation between the number of elements and the number of times that the number of rewriting rules sharply

It is evident from Fig.8 that sharp fluctuations in the number of rewriting rules occurred when the total number of elements was close to the maximum size of multisets. One of the reasons why this occurred is that Rule 2 tends to die out in a situation that the multisets are nearly saturated, because the right-hand side is bigger than the left-hand side, so that some room is needed for application, but there is not enough room 
around the situation. Because of this, the system lost some Rule $2 \mathrm{~s}$ which caused fluctuations of a number of other rules. This result exemplifies a situation where some rules cannot be applied sets off a chain reaction.

\section{Conclusion}

In this paper, we began our discussion by stating that it is efficient to form a cycle-oriented social system from the bottom up and that the belief that the pursuit of material circulation will lead to an ideal society is suspect. Then we described the modelling of an abstract cycle-oriented social system using ARMS. As the first step toward the construction of a framework for analysing sustainability, we showed that the number of rules corresponding to our actions depended on the complexity of the system and a complex system resulted in a large number of rules. However, we showed that a system having much higher complexity did not last for very long. From what has been discussed above, we can conclude that modelling and analysing the cycle-oriented social system using this method is an effective means of making the framework

We found that a system which has a high level of having great complexity did not last for very long and found the cause to be a sharp fluctuation of the number of rewriting rules in the simulation. Hence we need to consider restrictions in order to make the system having a high level of complexity sustainable to form a system that has diverse rules, as the next step.

\section{References}

[1] D.H.Meadows : The Limits to Growth : a report for the Club of Rome's project on the predicament of mankind, Universe Books (1972)

[2] Our Common Future, Oxford University Press (1987)

[3] A.Toffler : The Third Wave, Bantam Press (1991)

[4] K.Kakimoto, T.Shiose and T.Taura: Analyzing Sustainability of Circulatory System by Using Rewriting System; proceedings of EcoDesign 2001, IEEE, pp. 103-108 (2001)

[5] Y.Suzuki and H.Tanaka: Order Parameter for a Symbolic Chemical System; A life 4, MIT press, pp. 130-139 (1998)
[6] Y.Suzuki, Y.Fujiwara and H.Tanaka: Artificial Life and Rewriting System on Multisets, a class of P Systems; Lecture Notes on Computer Science, Springer Verlag, (to appear in)

[7] R. M. May: Stability and Complexity in Model Ecosystems; Princeton University Press (2001) 